\title{
Zahlen aus dem Kanton Schwyz
}

\section{Andreas Dummermuth}

lic. iur., MPA, Geschäftsleiter der Ausgleichskasse / IV-Stelle Schwyz

\author{
Konkrete Zahlen aus dem Kanton Schwyz zeigen, dass Eingliederungsmassnahmen \\ der IV nachhaltig erfolgreich sind. Sozialhilfe benötigt nur eine kleine Minderheit \\ der Versicherten, die den Anspruch auf eine IV-Rente verloren haben.
}

Im Kanton Schwyz sind zwei Drittel der Eingliederungsmassnahmen der Invalidenversicherung (IV) nachhaltig erfolgreich. Und 96 Prozent der Versicherten, die den Anspruch auf eine IV-Rente verloren haben, benötigen keine wirtschaftliche Sozialhilfe. Diese beiden Fakten sind Grundlagen für die Bewertung der sozialpolitischen Weichenstellungen durch den Gesetzgeber und ihrer Umsetzung durch die IV-Stellen.

\section{IV-Opfer und sozialer Tod?}

Dr. med. Doris Brühlmeier-Rosenthal schildert in ihrem Artikel [1] mit kräftigen Worten ihre Wahrnehmung im Bereich der IV. Als Leiter einer kantonalen IV-Stelle kann ich die Besorgnis und die sozialpolitischen Rügen einer praktizierenden Psychiaterin verstehen, ich betrachte jedoch mehrere Aussagen als Behauptungen, subjektive Wahrnehmungen und Wunschprojektionen. Frau Brühlmeier-Rosenthal blendet in ihrem Bericht die Tatsache aus, dass sich staatliche Organe schlicht und einfach an das in einem demokratischen Verfahren erlassene Recht halten müssen. Jeder Entscheid einer IV-Stelle kann deshalb auch immer von einem unabhängigen Gericht überprüft werden. Der vorliegende Artikel will einerseits mit Fokus auf die Eingliederung anhand einer Untersuchung aus dem Kanton Schwyz (154000 Bewohnerinnen und Bewohner) konkrete Zahlen und hoffentlich nachvollziehbare Auswertungen präsentieren. Andererseits möchte er, ebenfalls aufgrund einer konkreten Analyse in diesem Kanton, deren Resultate den gemachten Aussagen zum Thema «Verlagerung von IV-Rentnern in die Sozialhilfe» gegenüberstellen.

\section{Das Richtige richtig tun}

Jede Bürgerin und jeder Bürger, jede Prämienzahlerin und jeder Prämienzahler hat ein grosses Interesse daran: Wenn staatliche Organe etwas tun, dann soll es etwas bewirken. Dies gilt auch für die Sozialversicherer. In einem ersten Schritt muss jede IV-Leistung den rechtlichen Vorgaben entsprechen. Dazu klärt die IVStelle Schwyz den Sachverhalt ab. Das interdisziplinäre Team besteht aus Sozialversicherungsexperten, Eingliederungsfachleuten, Juristen und Verwaltungsangestellten. Unterstützt werden sie von den Ärztinnen und Ärzten des regionalen ärztlichen Dienstes (RAD). Besprechungen, eigene Abklärungen, Unterlagen der behandelnden Ärzte und der Arbeitgeber sowie Expertisen externer Fachpersonen dienen dazu, den Sachverhalt festzustellen. In den meisten Fällen und vor allem bei Eingliederungen besteht auch ein Kontakt zum Arbeitgeber und zum behandelnden Arzt. Sämtliche Leistungsprozesse der IV-Stelle Schwyz sind in einem Qualitäts-Management-System verbindlich und nachvollziehbar registriert. Rund 7000 Leistungsentscheide pro Jahr werden so gefällt. Und nochmals: Selbstverständlich unterliegen alle IV-Entscheide einer unabhängigen gerichtlichen Kontrolle.

In den meisten Fällen besteht auch ein Kontakt zum Arbeitgeber und zum behandelnden Arzt.

Aber nicht nur sachlich und rechtlich korrekt, sondern auch effizient und wirksam sollen die Leistungen gesprochen werden. Die Wirtschaftlichkeit der Arbeit der IV-Stellen ist gegeben: Die Verwaltungskosten der IV-Stellen sind mit 4,9 Prozent der Ausgaben (2016) dauernd und massiv tiefer als die Kosten der vergleichbaren Sozialversicherungszweige im Bereich der Arbeitslosen- oder Unfallversicherung. Und damit sind wir beim dritten Punkt: Wie steht es mit der Wirksamkeit? Erreicht die IV-Stelle die vom Gesetzgeber festgelegten Zielsetzungen? Und sind die Resultate nachhaltig? Auch diesen Fragen sind wir in Schwyz nachgegangen.

\section{Die berufliche Eingliederung ist nachhaltig erfolgreich}

Die Arbeit der IV-Stelle Schwyz hat einen nachhaltigen Erfolg. Dies können wir nachvollziehbar und trans- 
parent darlegen. Was heisst überhaupt Erfolg und was bedeutet Nachhaltigkeit? Diese beiden Begriffe müssen im technisch richtigen Kontext verstanden werden. Betrachten wir dafür den rechtlich verbindlichen Rahmen: die Bundesgesetzgebung. Die Invalidenversicherung hat gemäss Bundesgesetz (SR 831.10) einen dreifachen Auftrag. Artikel 1a des Invalidenversicherungsgesetzes (IVG) lautet:

Die Leistungen dieses Gesetzes sollen:

a. die Invalidität mit geeigneten, einfachen und zweckmässigen Eingliederungsmassnahmen verhindern, vermindern oder beheben;

$b$. die verbleibenden ökonomischen Folgen der Invalidität im Rahmen einer angemessenen Deckung des Existenzbedarfs ausgleichen;

c. zu einer eigenverantwortlichen und selbstbestimmten Lebensführung der betroffenen Versicherten beitragen

Wir fokussieren nachfolgend auf Art. 1a Buchstabe a: Geeignete, einfache und zweckmässige Eingliederungsmassnahmen sollen Invalidität verhindern. Die Invalidität stellt gemäss Gesetz eine voraussichtlich bleibende oder längere Zeit dauernde ganze oder teilweise Erwerbsunfähigkeit dar. Diese begründet dann einen Rentenanspruch. Die Umsetzung dieses Auftrags unterliegt dem Grundsatz der IV «Eingliederung vor Rente».

Jede berufliche Massnahme, die verhindert, dass es zu einer Rente kommt, ist ein Erfolg.

Innerhalb der Arbeitsprozesse der IV-Stelle können wir die entscheidenden Eckwerte «Eingliederungsmassnahmen" und "rentenbegründende Invalidität» eindeutig, klar und nachvollziehbar bestimmen.

\section{VERSICHERTE:}

Wir können sämtliche Leistungen auf die Einzelperson prüfen und auch langfristig vergleichen.

\section{RENTE:}

Sofern eine rentenbegründende Invalidität vorliegt, entsteht eine Teil- oder Vollrente der IV. Sie wird im Rentenregister eingetragen. Wir haben für die Auswertung also geprüft, ob zum Messzeitpunkt eine Teiloder eine Vollrente ausgerichtet wird oder nicht.

\section{EINGLIEDERUNG:}

Auf der anderen Seite haben wir geprüft, ob die IV-Stelle Schwyz eine berufliche Massnahme eingeleitet hat. Dies können wir ebenfalls aufgrund unserer seit dem Jahr 2000 elektronischen Fallführung im Einzelfall detailliert nachweisen. Jede Leistung der IV-Stelle wird genau und nach einem nationalen Standard codiert.

Um die Nachhaltigkeit zu messen, dürfen wir aber nicht die aktuellen Entscheide in einer Momentaufnahme zählen und bewerten. Es geht vielmehr darum, zu prüfen, wie sich die Entscheide der Vergangenheit längerfristig auswirken. Als Ausgangspunkt der aktuellen Untersuchung hat die IV-Stelle Schwyz die gesprochenen Massnahmen der Jahre 2011 und 2012 untersucht und geschaut, wie sich die Situation im August 2014 präsentierte. Erhält die Person, der im Jahr 2011 oder 2012 eine berufliche Massnahme zugesprochen wurde, im August 2014 nun eine IV-Rente oder nicht? Als «Erfolg» wurde definiert, dass die Person zum Analysezeitpunkt keine Rente bezieht. Gemäss dem Grundsatz «Eingliederung vor Rente» ist jede berufliche Massnahme, die verhindert, dass es zu einer Rente kommt, ein Erfolg. Und wenn mehrere Jahre nach der beruflichen Massnahme immer noch keine Rente notwendig ist, dann ist der Erfolg nachhaltig.

\section{Das Gesamtresultat für alle beruflichen Massnahmen}

Wenn wir die Gesamtheit der beruflichen Massnahmen betrachten, zeigt sich folgendes Bild: In den Jahren 2011 und 2012 hat die IV-Stelle Schwyz 445 berufliche Massnahmen an Versicherte gesprochen. 152 dieser Versicherten bezogen im August 2014 eine Teil- oder eine Vollrente. Der Eingliederungserfolg liegt somit bei gut 65 Prozent und im ähnlichen Rahmen wie bei der ersten Auswertung (535 Massnahmen in den Jahren 2007-2012, 535 Massnahmen und dann 190 Versicherte im August 2012 mit Teil- oder Vollrente).

Tabelle 1: Eingliederungserfolg 2012 und 2014

\begin{tabular}{lll}
\hline & August 2014 & August 2012 \\
\hline $\begin{array}{l}\text { Versicherte mit beruflichen } \\
\text { Massnahmen }\end{array}$ & 445 & 535 \\
\hline Davon mit Rente & 152 & 190 \\
\hline Eingliederungserfolg & $65,85 \%$ & $64,40 \%$
\end{tabular}

Das von uns gewählte Kriterium einer erfolgreichen Eingliederung ist sehr streng. Wir haben für die Auswertung als Erfolg einzig den vollumfänglichen Nichtbezug einer Rente definiert. Es gibt aber auch viele Versicherte, die nach beruflichen Massnahmen nur noch eine Teilrente benötigen. Sofern die beruflichen Massnahmen geholfen haben, dass die Teilrente tiefer ist als die Rente, die ohne die beruflichen Massnahmen notwendig war, ist auch dies ein Erfolg. Volkswirtschaftlich und sozialpolitisch ist eine Teilzeittätigkeit mit 
Teilrente nach Eingliederungsmassnahmen ganz klar als Erfolg zu betrachten.

Zudem gibt es Fälle, bei denen die IV-Stelle Schwyz berufliche Massnahmen spricht, obwohl die Wahrscheinlichkeit gross ist, dass die versicherte Person auch nach dem Abschluss der Eingliederungsmassnahmen eine IV-Rente benötigen wird. Wir sprechen hier von «Eingliederung in die Rente». Das ist zum Beispiel bei Personen mit Geburtsgebrechen, die später in einer geschützten Werkstätte arbeiten, der Fall. Auch dieser Ansatz ist sozialpolitisch und volkswirtschaftlich sinnvoll. Gemäss dem oben genannten Gesetzesartikel 1a Abs. 1 Buchstabe c ist eben auch die Verbesserung der Lebensführung ein gesetzlich verankertes Ziel der Sozialversicherung.

\section{Abschiebung in die Sozialhilfe?}

Die Interinstitutionelle Zusammenarbeit (IIZ) hat schweizweit und auch im Kanton Schwyz den Auftrag, die Zusammenarbeit zwischen den Organen der Arbeitslosenversicherung, der Berufs- und Studienberatung, der IV sowie der Sozialhilfe zu fördern. Betroffene Menschen mit einer Mehrfachproblematik sollen in Absprache aller Beteiligten und mit Nutzung aller möglichen Eingliederungsinstrumente den Weg zurück in die Arbeitswelt finden. Die IIZ-Koordinationsstelle des Kantons Schwyz beobachtet auch die Wechselwirkungen zwischen den verschiedenen Systemen. Politisch am brisantesten ist die Wechselwirkung zwischen der IV und der Sozialhilfe. Vielerorts und wiederholt wird behauptet, dass die IV-Stellen durch die Herabsetzung ihres Rentenbestandes mittels Rentenaufhebungen dafür sorgen, dass die Gemeinden als letztes Auffangnetz bei der wirtschaftlichen Sozialhilfe finanziell stärker belastet werden. Diese These der «Verschiebung» von IV-Rentnern in die Sozialhilfe wurde im Kanton Schwyz aufgrund aller konkreten Fälle in einem Zeitraum von fünf Jahren untersucht. Die IV-Stelle Schwyz hob in den Jahren 2009-2013 bei insgesamt 261 Versicherten die IV-Rente auf oder setzte sie herab. Die IIZ-Koordinationsstelle hat im Jahr 2014 die entsprechenden Daten der IV-Stelle Schwyz erhalten und konnte jeden einzelnen Fall den Bezügern für wirtschaftliche Sozialhilfe aller Schwyzer Gemeinden gegenüberstellen. Die Analyse aller Daten vor Ort im Mehrjahresvergleich zeigte, dass sich in zehn von 261 Fällen eine Verlagerung von der IV in die wirtschaftliche Sozialhilfe ergab. Das entspricht knapp vier Prozent der Fälle. Bei 96 Prozent der Rentenaufhebungen wurden die Gemeinden nicht leistungspflichtig.

\section{Die These der "Verschiebung» von IV-Rentnern} in die Sozialhilfe wurde im Kanton Schwyz untersucht.

Die These, dass IV-Rentenaufhebungen zu einer direkten Lastenverschiebung in die wirtschaftliche Sozialhilfe führen, erweist sich demnach im Kanton Schwyz als falsch. Die Untersuchung über fünf Jahre zeigt, dass sich eine Verschiebung nur in wenigen Fällen beobachten lässt.

Die vorliegende Untersuchung berücksichtigte nur jene Personen, die tatsächlich Anspruch auf eine IVRente hatten, welche dann aber herabgesetzt oder aufgehoben wurde.

\section{Schlüssel zum Erfolg}

Jede Eingliederung trägt auch die Unterschrift eines Arbeitgebers. Deshalb ist der gute Kontakt zur KMUgeprägten Schwyzer Wirtschaft für den Erfolg im Sinn der Versicherten entscheidend. Ebenso bedeutend ist aber auch die Partnerschaft mit den behandelnden Ärzten. Die nationale Informationsplattform www. pro-medico.ch ist dabei ein wichtiger Pfeiler. Noch wichtiger ist jedoch vor allem im Bereich der Eingliederung der direkte Kontakt zwischen den Eingliederungsfachleuten und dem betreuenden Arzt vor Ort. Auch hier kann man sagen: Es klappt immer besser, immer früher, immer verbindlicher mit der Bündelung der parallelen Interessen. Dafür gebührt den Ärzten ein aufrichtiger Dank. Erfolg entsteht schliesslich, wenn Fachleute Chancen erkennen und auch packen.

Brühlmeier-Rosenthal D: Soziales Elend nach Stopp oder
Verweigerung von IV-Renten. Schweiz Ärztezeitung. 2017;98(24):785-7. 